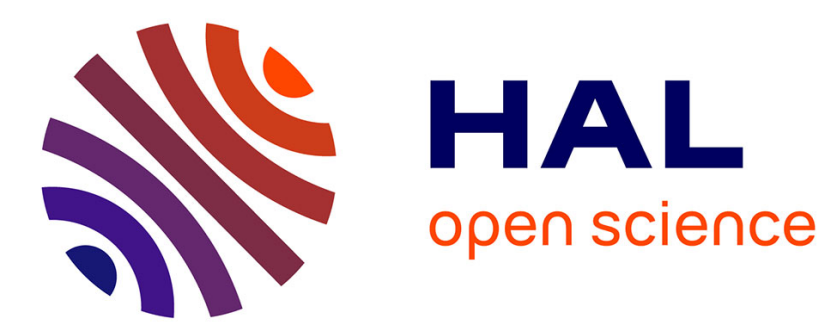

\title{
Capture of particles in soft porous media
}

Nicolas Louvet, Reinhard Höhler, Olivier Pitois

\section{To cite this version:}

Nicolas Louvet, Reinhard Höhler, Olivier Pitois. Capture of particles in soft porous media. Physical Review E: Statistical, Nonlinear, and Soft Matter Physics, 2010, 82, pp.041405. 10.1103/PhysRevE.82.041405 . hal-00786917

\section{HAL Id: hal-00786917 https://hal.science/hal-00786917}

Submitted on 20 Feb 2013

HAL is a multi-disciplinary open access archive for the deposit and dissemination of scientific research documents, whether they are published or not. The documents may come from teaching and research institutions in France or abroad, or from public or private research centers.
L'archive ouverte pluridisciplinaire HAL, est destinée au dépôt et à la diffusion de documents scientifiques de niveau recherche, publiés ou non, émanant des établissements d'enseignement et de recherche français ou étrangers, des laboratoires publics ou privés. 


\title{
Capture of particles in soft porous media
}

\author{
N. Louvet*, R. Höhler and O. Pitois \\ Université Paris Est, Laboratoire de Physique des Matériaux Divisés et Interfaces, FRE 3300, 5 bvd \\ Descartes, 77454 Marne la Vallée Cedex 2, France
}

\begin{abstract}
We investigate the capture of particles in soft porous media. Liquid foam constitutes a model system for such a study, allowing the radii of passage in the pore space to be tuned over several orders of magnitude by adjusting the liquid volume fraction. We show how particle capture is determined by the coupling of interstitial liquid flow and network deformation, and present a simple model of the capture process that shows good agreement with our experimental data.
\end{abstract}

DOI:

PACS numbers:

The capture of particles in porous media is widely encountered in technological processes of solids extraction from suspensions [1], as well as in natural phenomena [2]. Particle capture is intimately related to clogging of the pores and leads generally to harmful effects such as permeability reduction during water flooding operations in the petroleum industry or during recharge of aquifers. Clogging is a complex phenomenon involving a large number of parameters that have been partially decoupled in experiments conducted on model systems, such as beds of solid spheres [3], sieves [4] or solid channels [5]. The capture of particles is generally modelled in terms of size exclusion mechanisms, involving a single particle or particles aggregates $[4,5]$. In these studies, the effects of pores deformation were not taken into account, even though many porous materials can deform leading to complex elasto-hydrodynamic phenomena [6]. Moreover, in certain cases, the deformation of the porous network is very pronounced, as in unconsolidated soils or in intrinsically soft networks, such as biological tissues and blood vessels [7]. For soft systems, the pure size exclusion criterion is not able to predict the capture of particles and a more general theory is required.

Liquid foams can be considered as soft porous materials, exhibiting fine liquid channels between gas bubbles. The main asset of foams is their capacity to adjust dynamically the channel size over several orders of magnitude, in response to changes in interstitial liquid flow conditions [8, 9]. Moreover, foam channels can capture particles suspended in the liquid flowing through the gas bubbles, as reported for a broad range of systems [10]. This particle retention stabilizes foamed pastes, suspensions or emulsions, which have many applications in industry.

In the work presented here, we study particle capture in liquid foam, used as a soft porous medium model. The radii of passage in its pore space are tuned over a large range and the resulting capture mechanism is shown to depend on the coupling of interstitial liquid flow and network deformation. We present a simple model of this coupling that extends the basic size exclusion criterion to a more general one, showing good agreement with our experimental data.
The foaming solution contains $3 \mathrm{~g} / \mathrm{L}$ of TetradecylTrimethyl-Ammonium Bromide (TTAB) in distilled water. Density and shear viscosity of the bulk are respectively: $\rho=1000 \mathrm{~kg} / \mathrm{m}^{3}$ and $\eta=10^{-3} \mathrm{~Pa}$.s. The surface tension of the liquid/gas interface is $\gamma=38$ $\mathrm{mN} / \mathrm{m}$. The foam is made in a Perspex tube (length: $L=$ $60 \mathrm{~cm}$ and cross-sectional area: $S=25 \mathrm{~cm}^{2}$ ) by injecting slowly perfluorohexane saturated nitrogen gas into the aqueous solution with a syringe needle. This method yields foams with monodisperse bubbles, and the presence of perfluorohexane stops the bubble size evolution due to coarsening on the time scale of our measurements. Changing the syringe needle size allows us to vary the bubble radius $R$ in the range $0.5-2 \mathrm{~mm}$. Once the foam has filled the tube and after a free drainage step, a single spherical particle of radius $r_{s}$ is inserted and trapped at mid-height in the foam sample, using a thin glass capillary. The solid particles that we use are green fluorescent polystyrene spheres with a density $\rho_{s}=1050 \mathrm{~kg} / \mathrm{m}^{3}$. As $\rho_{s} / \rho=1.05$, the gravity force acting on particles will be neglected in the following. The sphere radius $r_{s}$, is chosen in the range from 25 to $170 \mu \mathrm{m}$ and it is sorted by video-microscopy to obtain a standard error less than $3 \%$. Then, the upper part of the foam is wetted by a constant liquid flow rate, $Q$, or equivalently a liquid flux, $V_{d}=Q / S$. Under these steady drainage conditions, the foam liquid fraction, $\varepsilon$, is uniform throughout the whole sample, and its value is related to $V_{d}$ through the foam permeability, $K$. Introducing the characteristic liquid velocity, $V^{*}=\rho g R^{2} / \eta$, and the dimensionless foam permeability, $R^{\circ}=K / R^{2}$, one can write:

$$
V_{d}=K(\varepsilon) V^{*} \text { or } \varepsilon=K^{\circ 0^{1}}\left(V_{d} / V^{*}\right)
$$

where $R^{2 / \sigma^{1}}$ is the inverse function of $R^{2 c}$. This latter quantity has been already determined for the foaming solution used in this work and it is published elsewhere [11]. Thus, in this experiment, tuning $V_{d}$ allows us to control the foam liquid fraction. For each measurement, 
$V_{d}$ is increased step by step, starting from a low value, until we observe that the trapped particle is ejected from the foam sample. Using this procedure we determine the relation between $V_{d}$ or $\varepsilon$, and the radius of capture $r_{\text {capt }}$ of the foam, defined as the radius $r_{s}$ of the particles ejected under these conditions. Such measurements are performed for foams made with three different bubble radii $(0.55 \mathrm{~mm}, 1.45 \mathrm{~mm}$ and $2.15 \mathrm{~mm}$ ) and for several particle radii. In Figure $1, r_{\text {capt }}$ is plotted as a function of $V_{d}$ and $R$. The radius of capture increases with both $V_{d}$ and $R$, and it varies over almost one order of magnitude. In the following, we interpret and we model these data.

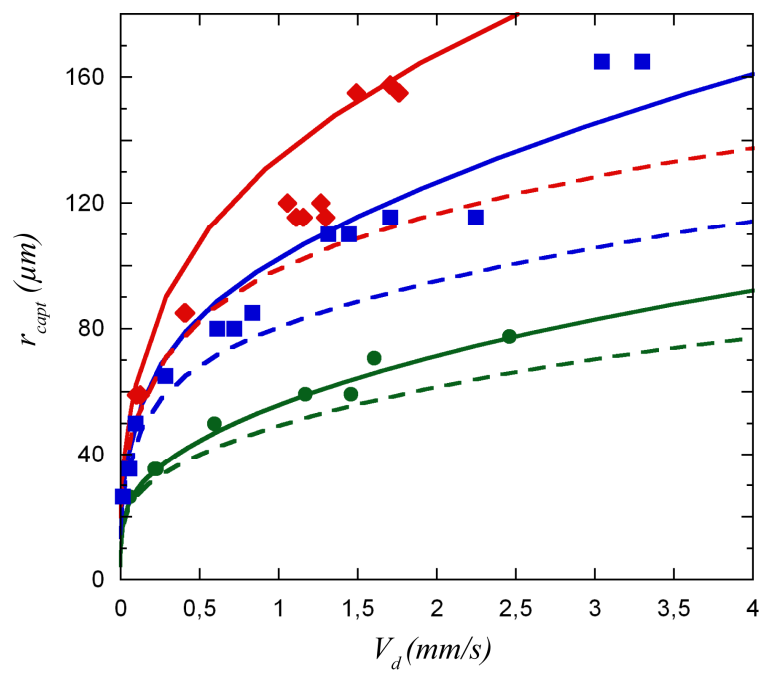

Fig. 1: Capture radius of foams vs. liquid flux. Bubble radius $R$ $(\bullet) 0.55 \mathrm{~mm},(\bullet) 1.45 \mathrm{~mm},(\bullet) 2.15 \mathrm{~mm}$. Full lines correspond to eq. (8). Dashed-line corresponds to eq. (4).

As a starting point, the radii of passage within the foam pore space have to be determined. The geometry of very wet foams resembles that of a sphere packing, and the radii of passage are: $r_{p}=\left(\frac{2 \sqrt{3}}{3}-1\right) R$. As the liquid fraction is reduced, bubbles flatten at their contacts and the radii of passage decrease (see Fig. 2). The geometry of the liquid network can be described as channels, the so-called Plateau borders, meeting 4 by 4 at nodes [8]. The size of these elements can be determined using the Surface Evolver software [12]. It is based on the principle that a foam with a given gas volume in each bubble has an equilibrium structure such that the interfacial energy is minimal. Previous work performed on liquid transport through foams has shown the relevance of a geometrical description based on monodisperse bubbles in a bcc arrangement [8]. Within the range of liquid fraction $0 \leq \varepsilon \leq 0.2$, the authors reported the evolution of the mean curvature of Plateau borders, which combines the two principal radii of curvature of their interface. For low liquid fractions, i.e. $\varepsilon \leq 0.02$, Plateau borders are essentially straight channels characterized by a single radius of curvature, $r$, and a corresponding radius of passage is given by the relation: $\varepsilon ; \delta_{1}\left(r_{p} / R\right)^{2}+\delta_{2}\left(r_{p} / R\right)^{3}, \quad$ with $\delta_{1} ; 13.8$ and $\delta_{2} ; 145$. Recently, however, it has been shown that the bcc structure is stable at low liquid fractions only, i.e. $0 \leq \varepsilon \leq 0.05$, and that for higher liquid fractions an energetically more favourable fcc structure is established throughout the foam sample [13,14].

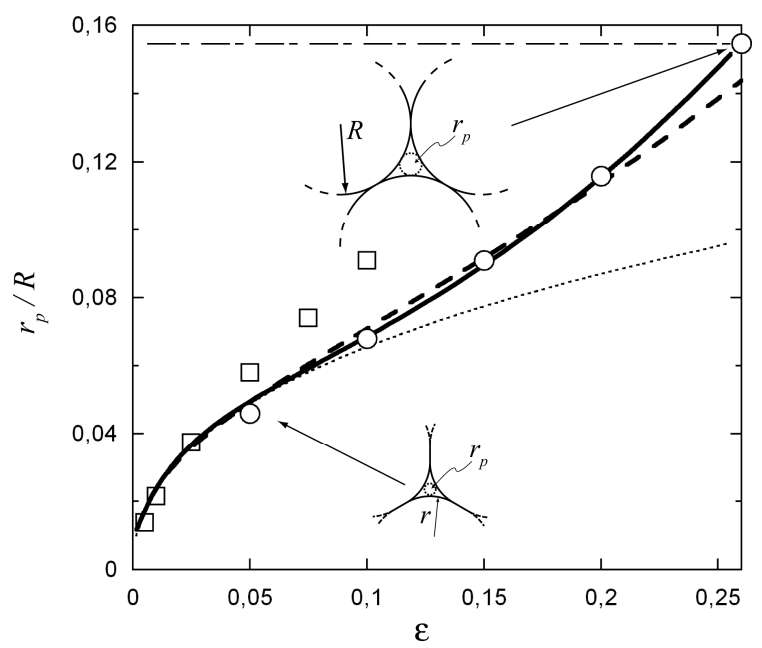

Fig. 2: Dimensionless radius of passage in foam as a function of liquid volume fraction. ( $\square$ ) and ( $\odot$ ) correspond to Surface Evolver calculations for bcc and fcc structures respectively. Full-line: eq.(2). Dashed-line: eq.(3). Dotted-line: values deduced from [8]. Sketches illustrate the evolution for the size of foam channels.

The monodisperse foams produced in our experiments look ordered when observed at the column wall. We therefore assume that depending on liquid fraction, they exhibit fcc or bcc polycrystalline structures, such as those observed in [13]. However, we expect the transition from fcc to bcc structures as a function of liquid fraction to be smoother than for fully crystallized samples. Using the Surface Evolver software, we simulate bcc structures for $\varepsilon \leq 0.1$ and fcc structures for $\varepsilon \geq 0.05$. In both cases, the radii of pore passage are determined directly from the simulated shapes of the Plateau borders (Fig. 2). We propose the following interpolation which is in good agreement with the bcc and fcc data in the full range $0 \leq \varepsilon \leq 0.26$ :

$$
\frac{r_{p}}{R}=\lambda_{0}(\varepsilon)=\frac{4 \cdot 10^{-3}+\varepsilon}{0.45+14.4 \varepsilon}+1.36 \varepsilon^{2}
$$

Note that our data are close to those deduced from [8] for $\varepsilon \leq 0.1$. However, a significant deviation is observed for higher liquid fractions because in [8] only the 
evolution for the mean radius of curvature in bcc structures is considered.

Results established for solid porous media offer an alternative way of estimating the radii of passage in foams. Johnson et al. have introduced an effective pore radius $\Lambda$ [15]: $\quad \Lambda=2 \int_{\text {pore }}|\stackrel{\mathrm{r}}{E}(\stackrel{\mathrm{r}}{r})|^{2} d V / \int_{\text {pore }}|\stackrel{\mathrm{r}}{E}(\stackrel{\mathrm{r}}{r})|^{2} d S$, which is a volume-to-surface pore ratio with a measure weighted by

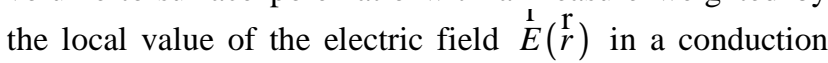
process. $\Lambda$ can be viewed as a dynamically weighted hydraulic radius. In contrast to the purely geometrical hydraulic radius, $r_{H}=(V / S)_{\text {pore }}$, this weighting eliminates contributions from the stagnant regions of the pore space that do not contribute significantly to transport. As demonstrated in [16], $\Lambda$ responds indeed directly to variations of the constrictions size in the pore space, i.e. the radius of passage of the pores: $r_{p}: \Lambda$. Interestingly, this length can be deduced from both permeability and conductivity measurements for a given porous medium: $\Lambda=m \cdot\left(8 K \sigma_{f} / \sigma\right)^{1 / 2}$, where $\sigma$ is the electrical conductivity of the sample, $\sigma_{f}$ is that of the pore fluid, and $m$ is a constant of order unity [17]. We propose to model the radius of passage in foam with the relation:

$$
r_{p}=m^{\prime} \cdot\left(8 K \sigma_{f} / \sigma\right)^{1 / 2}
$$

where $m^{\prime}$ is a constant. A complete set of data for the electrical conductivity of liquid foams and emulsions has recently been published and an empirical formula has been provided for the electrical conductivity as a function of liquid fraction [18]. Data for the permeability of foams with interfaces that mimic the solid walls of a porous medium where the liquid velocity vanishes at the pore surface, can be found in $[11,19]$. We describe these experimental results using the empirical expression: $K^{0} ; a b^{\varepsilon} \mathcal{E}^{c}$, with adjusted parameters: $a=2.8410^{-3}, \quad b=147$ and $c=1.9$. The determination of $r_{p}$ with eq.(3) requires the constant $m$ ' to be adjusted. We choose $m^{\prime}=0.73$, such that values provided by eq.(3) match those deduced from [8] in the dry foam limit $\varepsilon \leq 0.01$ where the radius of passage is precisely known. As illustrated in Fig. 2, eq.(3) and eq.(2) are in remarkable agreement, suggesting that the interpolation of bcc and fcc structures proposed here correctly describes how the channel size of monodisperse foams evolves in the full range of liquid fractions. This result also suggests that the modification of the channel network with liquid fraction, expressed in terms of channel length and node coordinance, is similar for ordered and disordered structures.

We now compare the radii of capture $r_{\text {capt }}$, measured as a function of $\varepsilon$, to the variation of $r_{p}$ with $\varepsilon$ described by eq (2). In view of this equation, a geometrical capture criterion $r_{\text {capt }}=r_{p}$, would predict:

$$
\frac{r_{\text {capt }}}{\lambda_{0} R}=1
$$

Using eqs (1) and (2), eq. (4) is plotted in Fig. 1. The comparison with the measured data shows that at low liquid flux, particle capture is indeed governed by a purely geometrical criterion. However, as the liquid flux increases, experimental data deviate from this simple criterion, especially for large bubbles. The transition between the captured state and the free state for particles in foam can be explained by the balance of viscous drag, which drives the particles through the foam channels, and capillary forces opposed to the particle motion at channel constrictions smaller than the particle diameter. We now describe the hydrodynamic breakthrough of the particle quantitatively, based on capillary and viscous forces.

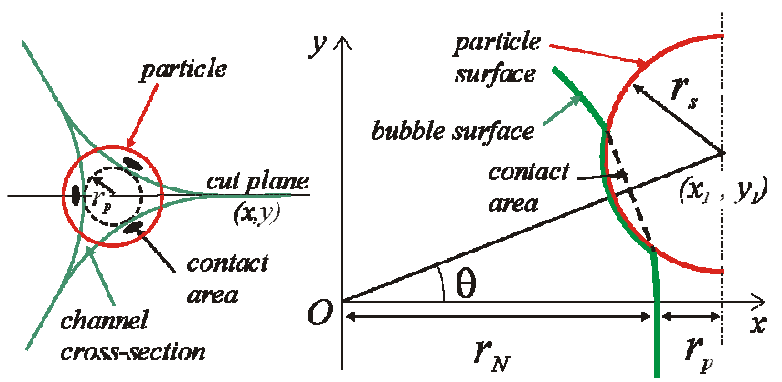

Fig. 3: Drawing of the particle at the entrance of a foam channel. Left: view along the channel axis. Right: view perpendicular to the channel axis

Let us consider a simple geometrical model for the foam pore structure: in the cut plane of Fig.3, the entrance of a channel is bounded by bubble interfaces with a radius of curvature $r_{N}$ given by $r_{N} / r_{p}=C$, where $\mathrm{C}$ is a geometrical constant. In the frame of Fig.3 (right), the position of a sphere centred at the entrance of the channel has dimensionless coordinates: $X_{1}=x_{1} / r_{p}=1+C$ and $Y_{1}=y_{1} / r_{p}$. As the sphere enters the channel, it touches the bubble surfaces for $Y_{1}=Y_{1}^{*} \quad$ given by $Y_{1}^{*}=\sqrt{\left(C+r_{s} / r_{p}\right)^{2}-(1+C)^{2}}$. Because the bubble surface is 'soft', this geometrical limit of the particle motion can be overpassed, i.e. $Y_{1}<Y_{1}^{*}$, and we assume that the resulting deformed bubble surface fits the sphere surface. Consequently, the capillary force acting on the sphere along the channel axis can be written:

$$
F_{c a p}=3 P_{c a p} S_{c} \sin (\theta)
$$


where $P_{c a p}$ is the bubble capillary pressure, $S_{c}$ is the projected contact area, $\theta=\tan ^{-1}\left[Y_{1} /(1+C)\right]$ is the polar angle (see Fig.3) and the coefficient 3 accounts for the three contacting bubbles that form the foam channel. Note that as $Y_{1}$ decreases, the contact area increases whereas $\theta$ decreases, so that the sphere experiences a maximum capillary force. Simple geometrical considerations show that the maximum capillary force is related to $r_{s} / r_{p}$ and $\mathrm{C}$ by:

$$
\frac{F_{\text {cap }, \text { max }}}{P_{\text {cap }} r_{p}^{2}} \approx \frac{19}{2} C^{-2 / 5}\left(r_{s} / r_{p}-1\right)^{1 / \beta}
$$

with $\beta=5 / 8$. As we consider small Reynolds numbers, the viscous drag force is modeled by:

$$
F_{v i s q}=\varsigma \eta r_{p} u_{l}
$$

where $\varsigma$ is the drag coefficient (which depends on the boundary condition at the channel surface) and $u_{l}=V_{d} / \varepsilon$ is the mean liquid velocity in the foam pore space. Assuming that capillary and viscous forces balance at the transition between the captured state and the free state we obtain a general capture criterion:

$$
\frac{r_{\text {capt }}}{r_{p}}=\frac{r_{\text {capt }}}{\lambda_{0} R}=1+\alpha f(\varepsilon) C a^{\beta}
$$

$C a=\eta V^{*} / \gamma=\rho g R^{2} / \gamma \quad$ is the capillary number, $\alpha=\left(2 \varsigma C^{2 / 5} / 19\right)^{\beta}$ is a constant considered as a fit parameter in the following. $f(\varepsilon)=\left(K^{2} d \varepsilon \beta_{\text {cap }} \lambda_{0}\right)^{\beta}$ is a function of the liquid fraction only, where $P_{c a p}^{o}=P_{c a p} /(\gamma / R)$ is given in [20] and $\tilde{K}$ in [11] for the same foaming solution we use in this study. Note that Eq.(8) includes the geometrical capture criterion given by eq.(4). In Fig.4, experimental data are compared to eq.(8) with $\alpha=11.6$. Both $\mathrm{C}$ and $\varsigma$ are required to determine $\alpha$ in eq.(8). An estimation based on Surface Evolver simulations yields $C \approx 20-30$, which correspond to 3 to 5 times the radius of curvature of the channel constriction [21]. A Recent experimental study of small particles settling in Plateau borders (formed with the same foaming solution as in this work) has provided a value for the drag coefficient $\varsigma \approx 73$, for the limit case $r_{s} / r_{p}$ close to unity [22]. Taking these values for $\mathrm{C}$ and $\varsigma$ we obtain $\alpha \approx 8$, which is in reasonable agreement with the fitted value. Fig.4 shows the effects of both liquid fraction and capillary number: (i) the so-called size exclusion criterion of solid filters, i.e. $r_{\text {capt }} / r_{p}=1$, is observed at small $\mathrm{Ca}$ and small $\varepsilon$; (ii) as both $\mathrm{Ca}$ and $\varepsilon$ increase, the ratio of viscous to capillary forces increases and the hydrodynamic breakthrough is more efficient. The second term of eq.(8) increases the radius of capture by $50 \%$ beyond its value for solid filters. For practical use, Fig. 1 shows the theoretical radius of capture as a function of $V_{d}$ and $\mathrm{R}$, obtained using eq.(1). This representation clearly shows the great benefit of using foams in filtering applications: a dynamical control of the radius of capture can be achieved by changing the flow rate of the filtered particle suspension.

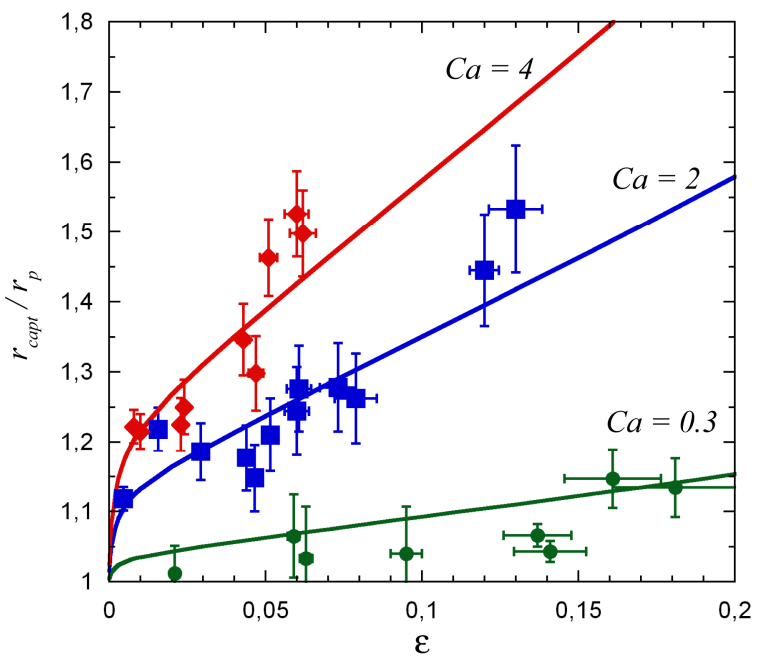

Fig. 4: Ratio of capture radius to passage radius as a function of liquid volume fraction. The Bubble radius $R$ is (•) $0.55 \mathrm{~mm},(\boldsymbol{\square}) 1.45 \mathrm{~mm},(\diamond) 2.15 \mathrm{~mm}$. The Full lines correspond to eq.(8).

To conclude, we have derived a criterion for particle capture in soft porous media, generalizing the classical size exclusion criterion of solid filters. We have shown that liquid foams are remarkable filtering materials, allowing the capture radius to be tuned dynamically over one order of magnitude. In this regard, our work opens a new way in the design of 'soft' filtering materials.

We gratefully acknowledge financial support from Agence Nationale de la Recherche (ANR-05-JCJC0234-01)

\section{References}

[1] C. Tien, Granular Filtration of Aerosols and Hydrosols (Butterworths, Boston, 1989); L. J. Zeman and A. L. Zydney, Microfiltration and Ultrafiltration: Principles and Applications (Marcel Dekker, New York, 1996)

[2] L. M McDowell-Boyer, J. R. Hunt, N. Sitar, Water Resour. Res. 22, 1901-1921 (1986)

[3] C. Ghidaglia et al., Phys. Rev. E 53, R3028 (1996)

[4] N. Roussel, Thi Lien Huong Nguyen and P. Coussot, Phys. Rev. Lett. 98, 114502 (2007) 
[5] H. M. Wyss et al., Phys. Rev. E 74, 061402 (2006); K. V. Sharp and R. J. Adrian, Microfluid. Nanofluid. 1, 376 (2005)

[6] H. F. Wang, Theory of Poroelasticity with Applications to Geomechanics and Hydrogeology (Princeton University Press, Princeton, NJ, 2000)

[7] C. Alberto Figueroa et al., Comput. Methods Appl. Mech. Engrg. 195, 5685-5706 (2006)

[8] S. A. Koehler et al., Langmuir 16, 6327 (2000).

[9] A. Saint-Jalmes et al., Phys. Rev. Lett. 98, 058303 (2007)

[10] F. Carn et al., Langmuir 14, 7847 (2009); K. Koczo et al., J. Colloid Interface Sci., 150, 492 (1992); Goyon et al., Phys. Rev. Lett. (2010); S. Guignot et al., Chem. Eng. Sci. 65, 2579 (2010); N. A. Bennani et al., Col. Surf. A 309, 7 (2007).

[11] E. Lorenceau et al., Eur. Phys. J. E 28, 293 (2009); F. Rouyer et al., Phys. Fluids (to be published)

[12] K. Brakke, Exp. Math. 1, 141 (1992).

[13] R. Höhler et al., Langmuir 24, 418 (2008).

[14] D. Weaire et al., Phys. Rev. Lett. 71, 2670 (1993); M.-D. Lacasse, G. S. Grest, D. Levine, Phys. Rev. E 54, 5436 (1996)

[15] D. L. Johnson et al., Phys. Rev. L, 57, 2564 (1986).

[16] S. Kosteck et al., Phys. Rev. B 45, 186 (1992).

[17] J. R. Banavar et al., Phys. Rev. B 35, 7283 (1987); D. Coelho et al., J. Colloid Interface Sci. 181, 169 (1996)

[18] K. Feitosa et al., Phys. Condens. Matter 17, 6301 (2005).

[19] O. Pitois et al., Langmuir 25, 97 (2009).

[20] S. Tcholakova et al., Phys. Rev. E 78, 011405 (2008).

[21] F. Rouyer, private communication

[22] O. Pitois et al., Phys. Fluids 21, 103304 (2009). 